\title{
Adaptive optics scanning laser ophthalmoscope imaging: technology update
}

This article was published in the following Dove Press journal:

Clinical Ophthalmology

26 April 2016

Number of times this article has been viewed

\section{David Merino \\ Pablo Loza-Alvarez}

The Institute of Photonic Sciences (ICFO), The Barcelona Institute of Science and Technology, Castelldefels, Barcelona, Spain
Correspondence: David Merino The Institute of Photonic Sciences (ICFO), Av Carl Friedrich Gauss, 3 , 08860 Castelldefels, Barcelona, Spain $\mathrm{Tel} / \mathrm{fax}+34935534002$

Email david.merino@icfo.es

\begin{abstract}
Adaptive optics (AO) retinal imaging has become very popular in the past few years, especially within the ophthalmic research community. Several different retinal techniques, such as fundus imaging cameras or optical coherence tomography systems, have been coupled with $\mathrm{AO}$ in order to produce impressive images showing individual cell mosaics over different layers of the in vivo human retina. The combination of $\mathrm{AO}$ with scanning laser ophthalmoscopy has been extensively used to generate impressive images of the human retina with unprecedented resolution, showing individual photoreceptor cells, retinal pigment epithelium cells, as well as microscopic capillary vessels, or the nerve fiber layer. Over the past few years, the technique has evolved to develop several different applications not only in the clinic but also in different animal models, thanks to technological developments in the field. These developments have specific applications to different fields of investigation, which are not limited to the study of retinal diseases but also to the understanding of the retinal function and vision science. This review is an attempt to summarize these developments in an understandable and brief manner in order to guide the reader into the possibilities that AO scanning laser ophthalmoscopy offers, as well as its limitations, which should be taken into account when planning on using it.
\end{abstract}

Keywords: high-resolution, in vivo retinal imaging, AOSLO

\section{Introduction}

Approximately 20 years have passed since the first adaptive optics (AO) system for retinal imaging was presented in $1997 .{ }^{1}$ Over this time, AO has gained popularity in the ophthalmic research community due to its capability to drastically improve the quality of retinal images by correcting the specific aberrations introduced by the cornea and lens of the individual's eye.

$\mathrm{AO}$ has been combined with techniques including scanning laser ophthalmoscopy (SLO), fundus cameras, and optical coherence tomography (OCT). ${ }^{1-5}$ Probably, the most significant achievement of AO-assisted imaging is the observation of the individual cell mosaics throughout the in vivo retina at different depths. ${ }^{1,6-10}$ Individual cone photoreceptor cells have been observed in the in vivo human retina without using $\mathrm{AO} ;{ }^{10}$ however, it is very difficult to observe these cells in the central fovea without $\mathrm{AO}$ because they are very densely packed. Using AO, not only cone but also rod photoreceptor structure has been studied in healthy subjects ${ }^{7,8}$ and in patients suffering from different retinal diseases. ${ }^{11-15}$ AO retinal imaging has helped to assess progression of the disease as well as response to novel treatments ${ }^{16}$ or even to observe spontaneous regeneration of photoreceptor outer segments. ${ }^{17}$ Figure 1 shows some adaptive optics scanning laser ophthalmoscopy (AOSLO) images from an in vivo healthy human retina, where the cone and rod photoreceptors can be observed. 


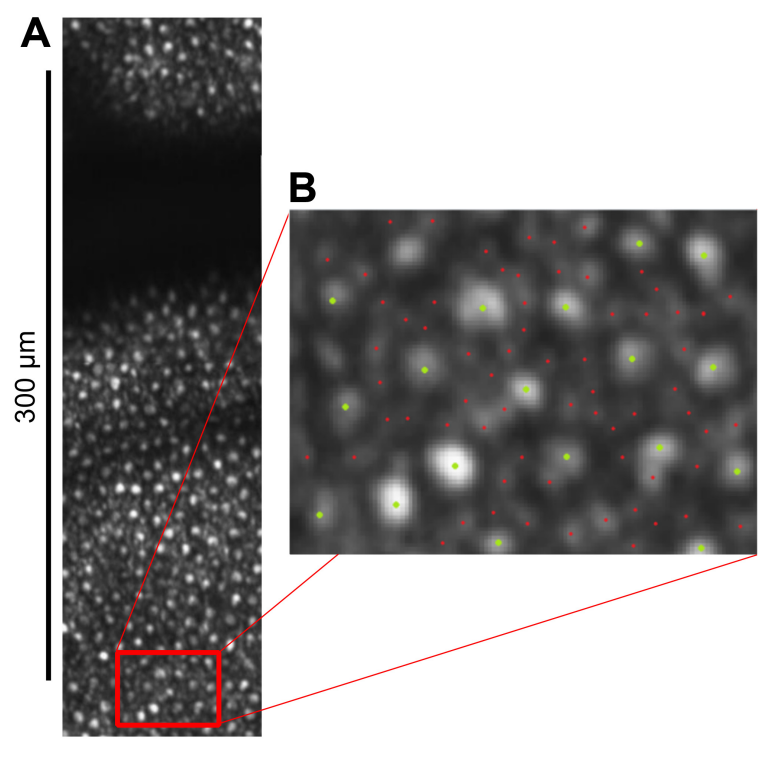

Figure I Image of the retina of a healthy subject acquired using AOSLO showing both cone and rod photoreceptors.

Notes: The image on the left is taken around $6.5^{\circ}$ measured down from the fovea (A). Image (B) shows a zoomed-in section of image (A); cones are marked in green and rods in red.

Abbreviation: AOSLO, adaptive optics scanning laser ophthalmoscopy.

This review focuses on AOSLO, which has been probably one of the most widespread AO-assisted retinal imaging techniques. In reflectance AOSLO, the light scattered back from the retina is used to generate an image by focusing it onto a pinhole that is placed in a plane conjugate to the layer of the retina that is being imaged. This provides the technique with optical sectioning capabilities. ${ }^{18-21}$ Using AO correction of the aberrations introduced by the eye, an increase in the resolution and contrast of AOSLO images compared to conventional SLO has been reported. ${ }^{20}$

This review briefly introduces recent advances in this technology, in an effort to guide the reader through different achievements that AOSLO has enabled over the years.

\section{Wavefront-sensing technology}

Usually, AO systems are composed of two main elements: a wavefront sensor (WS), which can measure the aberration that the subject's lens and cornea introduce in the imaging beam, and a wavefront corrector, usually a deformable mirror (DM), which can correct the aberration by physically changing its surface shape to match that of the aberration measured. As a result, AO can enable an aberration-free or a diffraction-limited system.

Generally, in AO retinal imaging systems, a small percentage of the light that is scattered back from the retina is sent to the WS to measure the ocular wave aberration. There are different WS approaches implemented in the eye, such as laser ray tracing and pyramid or curvature WS. ${ }^{22-24}$ However, probably due to its simplicity and robustness, one of the most widespread WS types for AO retinal imaging is the ShackHartmann WS. ${ }^{25}$ In this type of devices, the light propagates through a lenslet array, and each of these lenslets focuses the light onto a spot on a charge-coupled device (CCD) camera. Aberrations on the beam change the position where these lenslets focus the light, and this displacement of the spots on the CCD is used to reconstruct the wavefront.

Probably, one of the main applications of AO may be the study of degenerative diseases that often affect elderly patients. It is common that these patients present cataracts or media that are not too transparent due to their advanced age. This can produce an increased background on the ShackHartmann WS signal due to the scattering of light, limiting its performance. Sometimes the disease that affects the patient can produce a reduction in the reflectivity of the retina, and this can also translate into limited performance of the WS.

It is possible to implement an AO retinal imaging system eliminating the WS, thus avoiding its poor performance. Furthermore, all the light scattered back from the eye can be used to generate the images and increase the signal-to-noise ratio (SNR). In order to implement a wavefront sensorless AO system, a metric that gives information on the quality of the image is needed. In the case of a confocal system, a simple quantity, such as the overall intensity in the image, may be sufficient. ${ }^{26}$ However, other more complicated metrics can be used, eg, different definitions of image sharpness. ${ }^{27}$ Once a metric has been chosen, an iterative algorithm is used to determine the shape of the wavefront corrector that maximizes this metric. There are different algorithms that can be used to optimize it, such as random searches or genetic algorithms, which have been implemented before in AO microscopy. ${ }^{28}$

Wavefront sensorless AO has been reported in AOSLO, choosing the overall image intensity as the image quality metric and iterative stochastic parallel gradient descent as the algorithm to optimize it. ${ }^{29}$ The main drawback of this kind of systems is that it usually takes a considerable time for the iterative algorithm to converge to an optimal correction. This may become a problem when imaging the in vivo retina, where eye motion and blinking may be challenging. Recent methodology using ultrahigh-speed graphicsprocessing-unit-based platforms combined with a specific algorithm has allowed for much faster implementations of this technique that has been applied to generate in vivo en-face OCT images of the human photoreceptor mosaics at different eccentricities. ${ }^{30}$ 


\section{DM technology}

At the time when the first applications of AO for retinal imaging were implemented, DMs had, in general, limited stroke values and limited number of actuators that were fit into relatively large pupil sizes. These limitations made it difficult for devices available at the time to accurately reproduce the wavefront measured by the WS. One of the first solutions to this problem is to precompensate the aberration using trial lenses that correct the lower-order aberrations of the subject. ${ }^{31}$ This way, the DM corrects only the higher-order aberrations, which in general require lower stroke. However, this approach requires manually changing the graduation of trial lenses for each patient, which can be a tedious process.

Another strategy that has been followed to obtain the best results from the technology available has been to use more than one DM with different capabilities that complement each other. In general, this strategy for the correction of aberration is referred to as the woofer-tweeter configuration, in which two DMs are used. ${ }^{32,33}$ One of them, the woofer, presents high stroke; however, since it is usually equipped with a low number of actuators, it can only reproduce smoother surfaces with low spatial frequencies. This mirror is commonly related to the correction of lower-order aberrations, such as defocus and astigmatism, which are quite stable throughout the imaging process. The second mirror, commonly referred to as the tweeter, usually has a lower stroke but a large number of actuators and, therefore, can reproduce surfaces with high spatial frequencies. The combination of these two DMs has allowed for the automatic correction of wavefront with large range and higher-order aberrations. These two mirrors are implemented together in the same AO loop system where some complex algorithms are required in order to obtain optimum results. ${ }^{32,33}$

Currently, DMs with high stroke values (up to $\sim 100 \mu \mathrm{m}$ ) and hundreds of actuators that are squeezed in small pupil sizes $(\sim 7 \mathrm{~mm})$ can be found in the market. By using this new technology of DMs, the design of AOSLO systems can become simpler and more versatile.

\section{Optical design}

Traditionally, not only SLO but also OCT systems tend to avoid the use of lenses in their design because of the spurious reflections that may generate on their surfaces. In AOSLO systems, these reflections may affect not only the quality of the images but also the performance of the WS, since the amount of light that is scattered back from the retina can be comparable to that reflected from the optics of the system, even if they are appropriately antireflection coated. ${ }^{34}$ To avoid these reflections from relay optics, offaxis spherical mirrors are used rather than lenses. However, this approach introduces astigmatism, which decreases the quality of the image of the pupil of the eye that is created on the WS and has an impact on the performance of the AO system. By taking into account not only the quality of the beam focused onto the retina but also the quality of this pupil image on the WS, the quality of the AO correction achieved is also improved. A straightforward way to reduce astigmatism is to use spherical mirrors with long focal lengths, reducing the off-axis angle. However, this may increase the overall size of the system. ${ }^{35}$ Another way to minimize astigmatism is to adopt optical designs that are not confined to just one single plane. By alternating the angle of the reflection of the beam of these mirrors from the horizontal to vertical directions, astigmatism can be minimized. ${ }^{35,36}$ AOSLO systems designed using this concept have reported the capability of observation of not only individual cone photoreceptors but also rod photoreceptors, which have a size that is close to the limit of the resolution of the eye. These were observed not only in healthy subjects but also in patients suffering from several different retinal diseases. ${ }^{7}$ Figure 2 shows some AOSLO images of healthy and diseased retinas, where both cone and rod photoreceptors can be observed.

Despite the larger size of these systems, another advantage of the use of spherical mirrors instead of lenses is that mirrors do not present chromatic aberration. Thus, several channels using different excitation and imaging wavelengths can be implemented simultaneously on the same optical system. For example, a dedicated light source can be used in order to image and detect aberrations, and also another to excite autofluorescence (AF) or fluorescence from exogenous and endogenous agents, ${ }^{37}$ as it is described in more detail in the "Fluorescence and AF imaging" section. These different channels can be implemented along with the conventional reflectance AOSLO imaging system. In this way, different retinal imaging modalities can be implemented in one system, and complementary information extracted from each one can be used to better characterize the retina.

Recently, a different approach on the implementation of AOSLO systems has been reported in which light polarization is used to avoid spurious reflections in optical setups that use lenses. ${ }^{38}$ In this approach, a quarter wave plate is placed in front of the cornea, so that the light that is scattered back from the retina conserving its polarization state changes its angle of the polarization to be $90^{\circ}$ after passing twice through the plate. A polarizing beam splitter is used to discriminate 


\section{A}

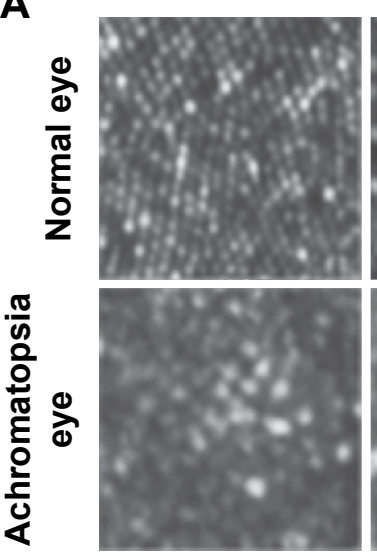

$0.5^{\circ}$

B
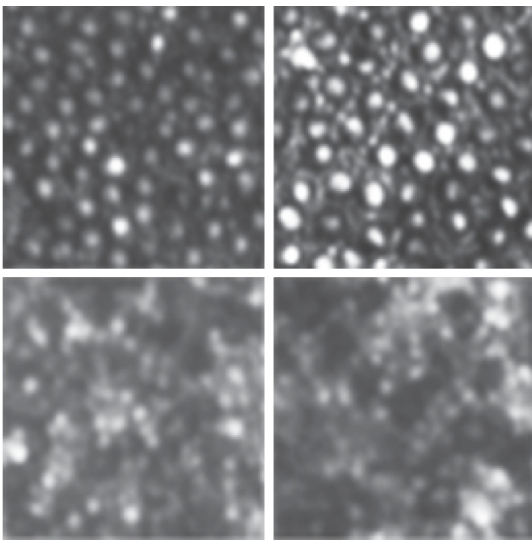

$4^{\circ}$

Eccentricity

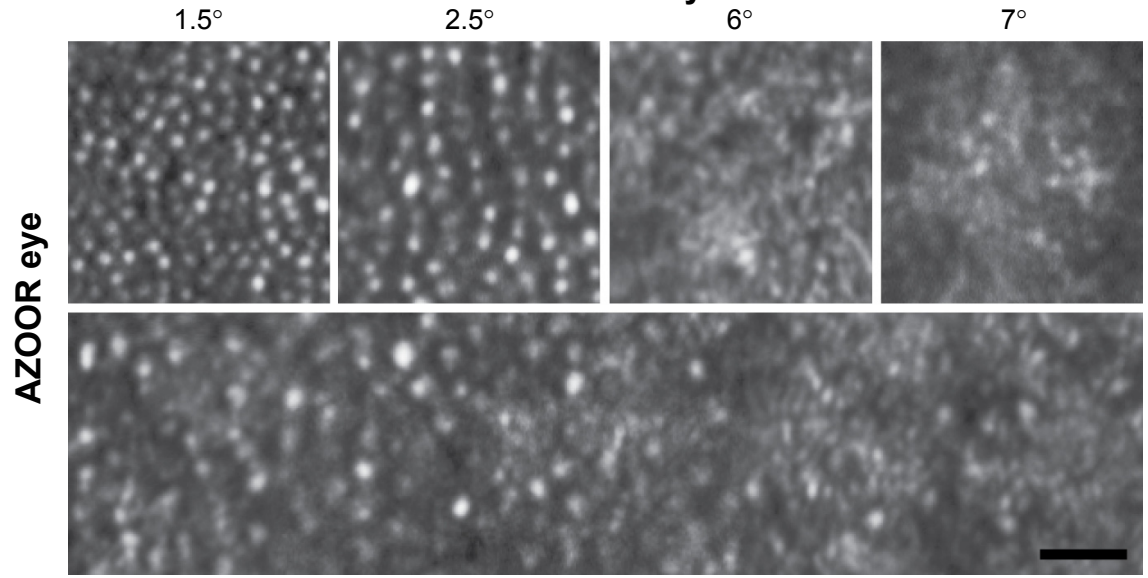

$4.5^{\circ}$

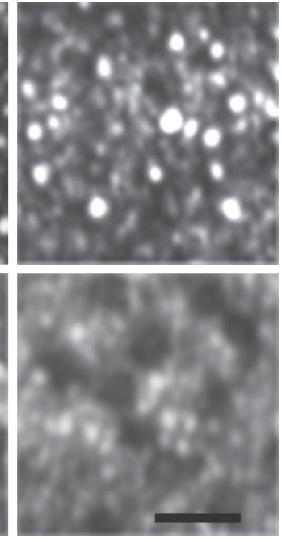

$11^{\circ}$

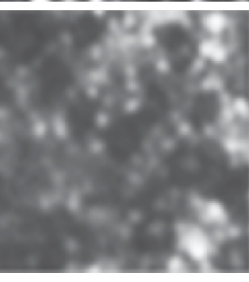

$7^{\circ}$ $7^{\circ}$

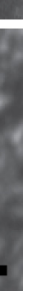

Figure 2 Cone and rod photoreceptor mosaic for healthy and diseased in vivo retinas.

Notes: (A) AOSLO retinal images at different eccentricities of a healthy subject compared to those from an achromatopsia patient. Scale bar is $20 \mu \mathrm{m}$. (B) AOSLO images of an AZOOR patient. The retina seems normal up to an eccentricity of 4.5, where a lesion is found, and extends up to 7 . In the lesion, cones are not observed, although rods seem present. Scale bar is $20 \mu \mathrm{m}$. Copyright @20I I. Adapted from Merino D, Duncan JL, Tiruveedhula P, Roorda A. Observation of cone and rod photoreceptors in normal subjects and patients using a new generation adaptive optics scanning laser ophthalmoscope. Biomed Opt Express. 201 I;2(8):2189-2201, with permission from The Optical Society.'2

Abbreviations: AOSLO, adaptive optics scanning laser ophthalmoscopy; AZOOR, acute zonal occult outer retinopathy.

the light scattered from the lenses, which does not change its polarization. The main advantage of this technique is that lenses do not present astigmatism as spherical mirrors do, and the size of the system can be drastically reduced without a great impact on the image quality. The performance of lenses and retarding plates is dependent on the wavelength of the light beam, and this approach may not be suitable for the implementation of imaging channels using different light wavelengths. Furthermore, fluorescence and AF signal does not preserve the polarization of the excitation beam, and the polarization cannot be used to select retinal fluorescence signal. Nonetheless, this technique has been successfully combined with OCT to simultaneously produce SLO and OCT images with pixel-to-pixel correspondence. This approach has been used to image individual photoreceptor cells throughout the retina ${ }^{39}$ and also the retinal vasculature. ${ }^{40}$
These achievements are further discussed in the "Simultaneous AOSLO and OCT" section.

\section{Confocal and nonconfocal imaging}

One of the main advantages of AOSLO is the fact that it is based on a confocal configuration. This translates into the capability for optical sectioning to generate images of planes at different depths in the retina. ${ }^{20,34}$

As shown in Figures 1 and 2, reflectance AOSLO has allowed for the observation of the photoreceptor mosaics in the living retina, since these cells present quite high scattering characteristics compared to other layers in the retina. Furthermore, the optical sectioning capabilities of AOSLO have been used to study elevations within the photoreceptor layer, which can be relevant to different diseases, such as age-related macular degeneration, where the evolution of photoreceptor 
mosaic has been studied over time, ${ }^{41}$ or in different types of elevations associated with the disease. . $^{13,41,42}$

Other microscopic features that lay in other layers of the retina have also been observed thanks to optical sectioning capabilities of AOSLO. For example, images of the nerve fiber layer (NFL) have been used to assess abnormalities in patients with different retinal diseases, correlating these results with frequency-domain OCT. ${ }^{43-45}$ Also, the Henle fiber layer has been observed in patients with diseases that may affect the laminar arrangement of the retinal layers. ${ }^{46}$

Another popular application of AOSLO is blood vessel and capillary imaging. Although the observation of blood vessels in layers between photoreceptors and NFLs has been reported since the first implementation of AOSLO was reported, ${ }^{20,21}$ image analysis techniques have allowed for the observation of small capillary vessels around the fovea. In these experiments, images of the photoreceptor layer are acquired over a certain period of time. Due to the absorption of light with long wavelengths by blood and blood vessels themselves, a variation in time on the contrast of AOSLO images is observed. This variation has been used to enhance the contrast of parafoveal capillary images and also to characterize the speed of blood components, such as leukocytes or erythrocytes, through them. ${ }^{47-50}$ This method has allowed for the whole mapping of the parafoveal capillary network and the precise determination of the foveal avascular zone. ${ }^{47-49,51}$ Figure 3 shows the capillary bed around the foveal avascular zone acquired using temporal changes of reflectance as contrast source. It is important to notice here that no exogenous contrast agents are needed in this technique, and since it is noninvasive it has been extensively

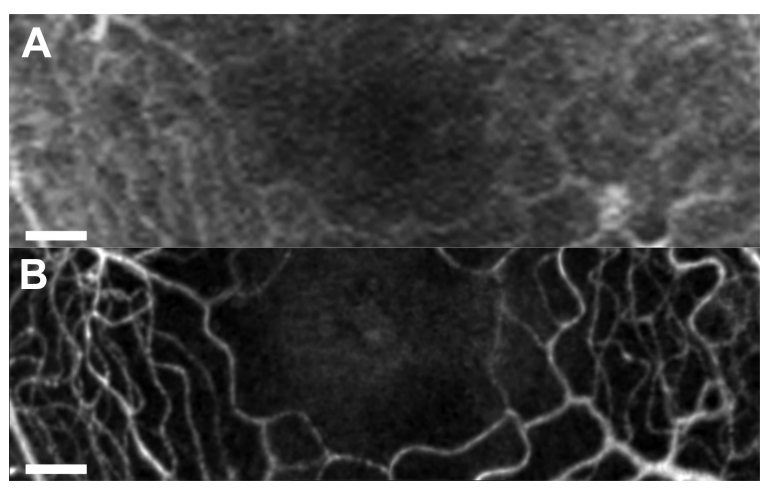

Figure 3 Capillary vessel structure around the foveal avascular zone.

Notes: (A) Traditional fluorescence angiography image acquired using Spectralis HRA (Heidelberg Engineering, Heidelberg, Germany). (B) Image of the same subject obtained by using time changes of reflectivity as a source for contrast. Courtesy of Dr Johnny Tam. Scale bar is $100 \mu \mathrm{m}$.

Abbreviation: HRA, Heidelberg retinal angiograph. used to characterize changes in retinal vasculature related to diabetes and diabetic retinopathy. ${ }^{52,53}$

Another microscopic feature observed in the in vivo human retina using AOSLO is the cell mosaic in the retinal pigment epithelium (RPE). This was first observed in areas where the photoreceptors have disappeared due to different retinal diseases, such as cone-rod dystrophy and bilateral progressive maculopathy. ${ }^{54}$ However, the observation of RPE cells in healthy subjects has been challenging because this layer lays just posterior to the photoreceptor layer. Light scattered from the RPE layer is masked by the strong scattering signal from the photoreceptors. A similar situation is also observed in the small vessels that lay just behind the NFL; the highly scattering properties of this layer make it difficult to observe less scattering capillaries. Recent studies using forward scattered photons imaging have extended into imaging of the in vivo human retina using AOSLO the concepts of nonconfocal imaging previously developed in microscopy. In forward scattered photons imaging, the confocal pinhole is moved away from the optical axis of the system and also increased in size. In confocal imaging systems, this pinhole usually allows for the in-focus photons to reach the detector; however, when it is displaced, these in-focus photons are blocked, and only the photons that have been scattered multiple times can reach the detector. For this reason, this technique is called nonconfocal imaging. Different implementations of nonconfocal imaging have been reported, in which the confocal pinhole has been replaced by different geometrical shapes, including a circle, an annulus, a circle with a filament across the center, and a circle combined with a knife edge. These configurations were tested on the same imaging system in order to determine the aperture configuration that optimizes contrast, specifically to study retinal vascular structure. The conclusion of this work is that this optimal configuration corresponds to the split-detection scheme. ${ }^{55}$ In this technique, the nonconfocal aperture used is a circle that is divided into two symmetrical areas by a filament that crosses it through its center. The photons reaching each of these areas are sent to different detectors. Images are then generated by measuring the differences in intensity between the two detectors, which are divided by their sum. Using nonconfocal imaging techniques, an increase in the contrast of features that were masked by the effect of more scattering structures in the confocal image has been observed, such as the case of RPE cells in healthy retinas masked by the effect of neighboring photoreceptor cells, or the case of capillary vessels that are positioned close to the NFL. ${ }^{56,57}$ Figure 4A shows an image 

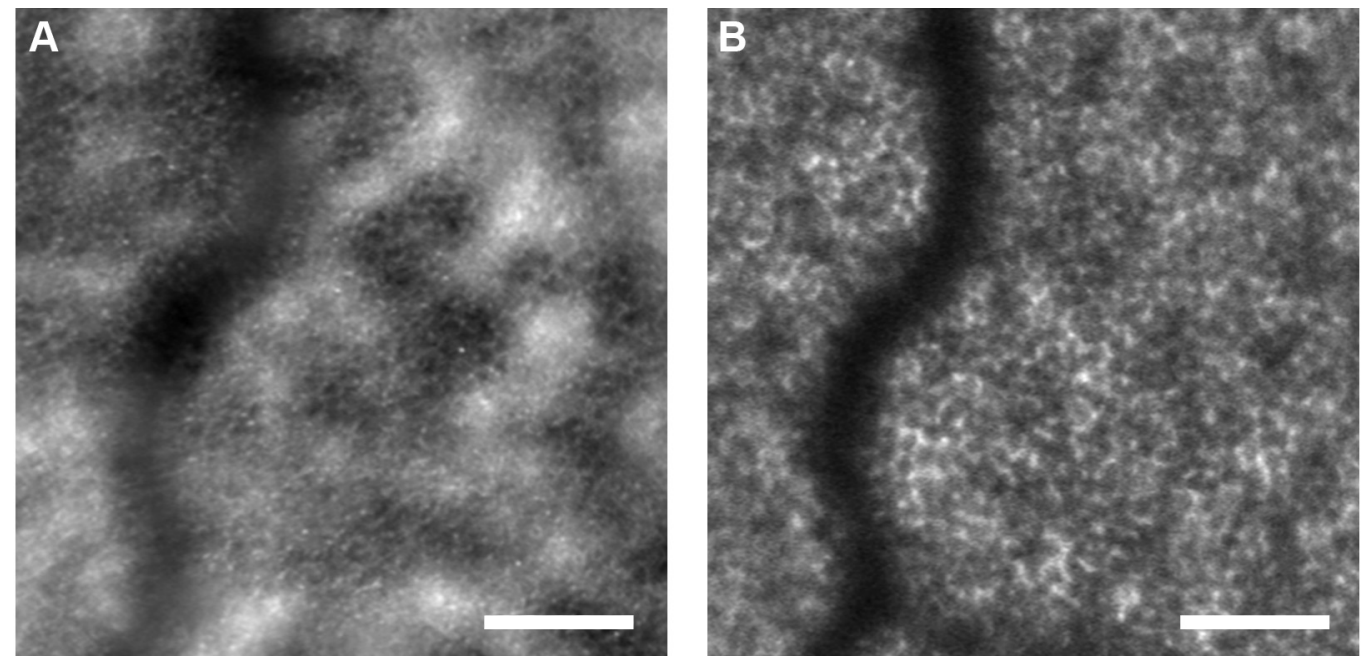

Figure 4 Comparison of autofluorescence to dark-field RPE imaging in a healthy subject at $3^{\circ}$ superior and $9^{\circ}$ temporal from fixation.

Notes: (A) Dark-field image. (B) Autofluorescence images collected using $565 \mathrm{~nm}$ excitation and $625 \pm 45 \mathrm{~nm}$ emission. The scale bar is $100 \mu \mathrm{m}$. Copyright @ 2013 . Reprinted from Scoles D, Sulai YN, Dubra A. In vivo dark-field imaging of the retinal pigment epithelium cell mosaic. Biomed Opt Express. 20I3;4(9): 17I0-1723, with permission from The Optical Society. ${ }^{57}$

Abbreviation: RPE, retinal pigment epithelium.

of the RPE cell mosaic in a healthy subject acquired using split-detection AOSLO.

Despite the challenges in understanding the source of contrast of these images, the use of this split detection has provided impressive images and has recently been applied to image the in vivo mice retina. The mouse eye has a high numerical aperture compared to that of human subjects, which translate into images with better resolution. Under these conditions, the observation of individual retinal cells has been achieved not only in the photoreceptor layer but also in the most transparent layers of the retina, such as the outer nuclear layer. In addition, horizontal cells have been observed in the outer plexiform layer without the use of contrast agents for the first time in vivo using split detection. ${ }^{58}$ Figure 5 shows some images from different layers of the mouse retina acquired using split-detection AOSLO.

\section{Fluorescence and AF imaging}

$\mathrm{AF}$ imaging is a tool very commonly used in ophthalmology, and it is applied to observe the RPE of patients. ${ }^{59}$ An excitation beam in the blue region of the spectrum is used to excite intrinsic fluorescence on the lipofuscin granules that accumulate in the cytoplasm of RPE cells through the processes of the visual cycle and phagocytosis. As mentioned earlier, the use of different excitation and detection wavelengths can be easily implemented in AOSLO systems that have been built using spherical mirrors, since they do not present chromatic aberration. This feature has been exploited in order to combine fluorescence and $\mathrm{AF}$ imaging with conventional reflectance AOSLO imaging.
AF AOSLO systems have allowed for the observation of the RPE cell mosaic in healthy subjects. As detailed in the "Confocal and nonconfocal imaging" section, the observation of RPE cells has been challenging in healthy subjects due to their proximity to the photoreceptor layer. ${ }^{60,61}$ Even when using nonconfocal imaging, contrast in RPE cell mosaic can be low, ${ }^{57}$ as it is shown in Figure 4A. By using optical filters to block scattered light from the photoreceptors and only allowing AF signal from RPE cells to reach the detector, these have been observed thanks to the high resolution capabilities of AF AOSLO. ${ }^{60}$ Figure 4B is an AF AOSLO image acquired in a healthy subject showing the RPE cell mosaic. Furthermore, by automatically correcting for chromatic aberration introduced by the eye itself on the images acquired using AF AOSLO, RPE cells have also been observed in patients suffering from age-related macular degeneration. ${ }^{61}$

Fluorescence imaging of the retina has not been limited to intrinsic tissue fluorescence but also contrast agents have been used to image the human retina in vivo. In particular, intravenous and oral fluorescein have been used to visualize retinal capillaries in animal models and also in healthy human subjects. ${ }^{62,63}$ The results show that all retinal capillary beds can be imaged using clinically accepted fluorescein dosages at safe light levels. These results show that fluorescein signal in the retinal capillaries peaks at different times depending on whether it is administered orally or intravenously. Although specific peak and disappearance times vary across individuals, in general, orally administered fluorescein can be imaged for a longer period of time in the retina; however, fluorescence intensity also peaks at a later time when 


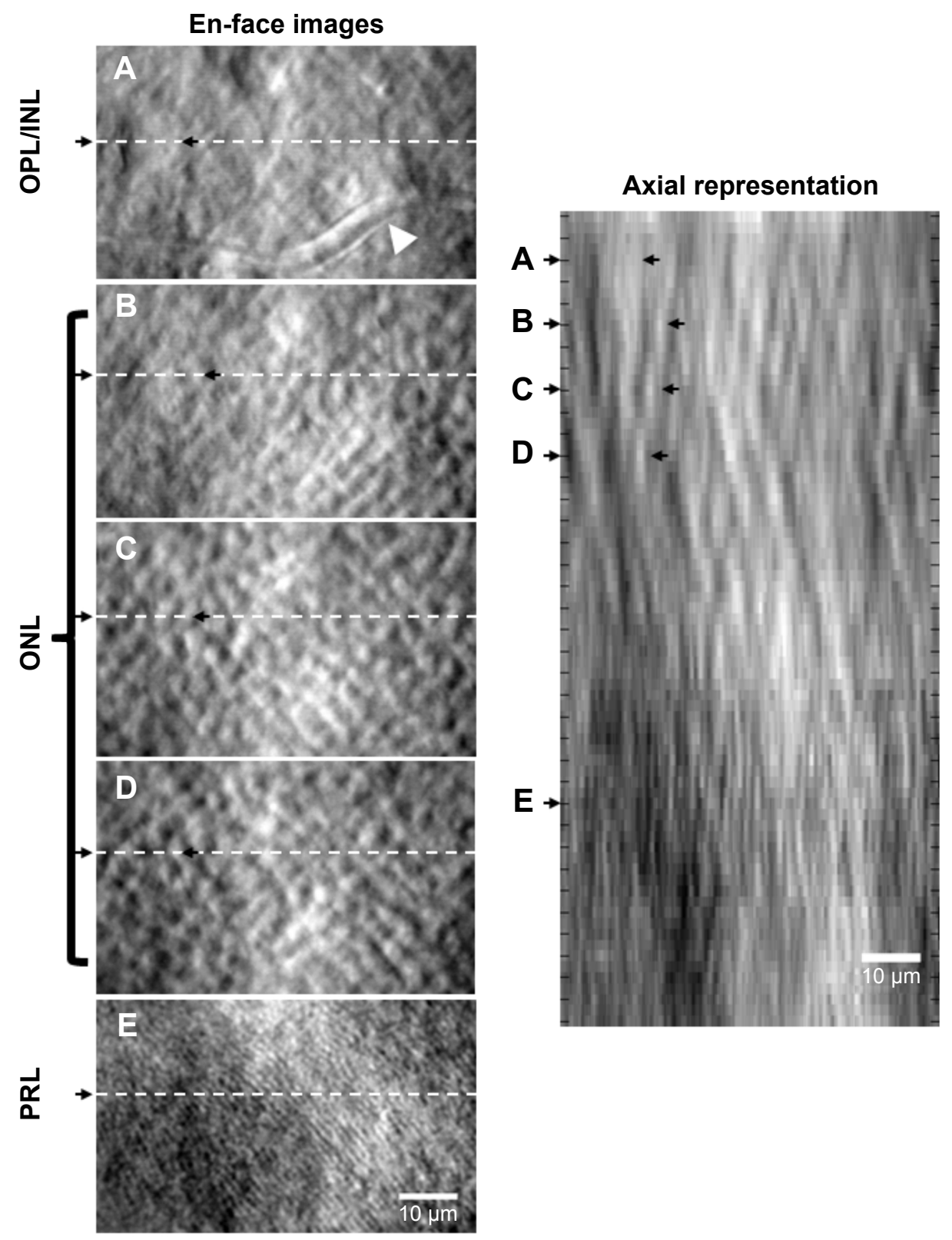

Figure 5 Images of a murine retina acquired by means of split detection AOSLO.

Notes: The left panel shows a through focus sequence of en-face images starting at the OPL and finishing slightly below the photoreceptor layer. The image on the right is an axial reconstruction of the retina along the optical axis generated using 38 different en-face images. The depths at which images (A-E) are acquired are marked on the image on the right. (A) The boundary of the OPL and INL is anatomically confirmed by the presence of the deepest layer of retinal vessels (white arrowhead). Images (B-D) represent different depths on the retina. The exact positions on the retina where (B-D) can be found are marked on the axial reconstruction on the right panel. The multilayered structure of the photoreceptor somata is captured in en-face images from different focus depths within the ONL. (E) The finer structure of the monolayer of photoreceptor distal segments in the PRL. The right panel shows the axial rendering of 38 en-face images separated by equal dioptric steps as measured by the AO control software. Tick marks in the image represent data from a single imaged plane. Lines in between represent linearly interpolated pixels in the $x-z$ representation. Distinct features in the en-face images (black arrows) are colocalized in the axial representation. Copyright @20I5. Reprinted from Guevara-Torres A, Williams DR, Schallek JB. Imaging translucent cell bodies in the living mouse retina without contrast agents. Biomed Opt Express. 2015;6(6):2106-21 19, with permission from The Optical Society. ${ }^{58}$ Abbreviations: AO, adaptive optics; AOSLO, adaptive optics scanning laser ophthalmoscopy; INL, inner nuclear layer; ONL, outer nuclear layer; OPL, outer plexiform layer; PRL, photoreceptor layer.

compared to intravenous administration. AOSLO fluorescein angiography has been applied to image microaneurysms in patients with vascular retinopathies, such as diabetic or hypertensive retinopathy, and branch and central retinal vein occlusion. ${ }^{64}$

$\mathrm{AF}$ and fluorescence imaging using AOSLO has raised some concerns related to subjects' discomfort and safety. As it is explained in more detail in the "Safety issues" section, changes in the AF signal acquired using AOSLO from the retina have been observed in animal models and also in humans when using this technique, even when the intensity of the illumination beams was below the limits set by safety standards. ${ }^{65-68}$ Reports comparing the results acquired using fluorescence and AF AOSLO with forward scattered photons 
imaging seem to show that nonconfocal imaging techniques, such as split detection, may be suitable alternatives to fluorescence and AF not only to image the human RPE cell mosaic $^{57}$ (as it is shown in Figure 4) but also to acquire images of the capillary network in the human retina. ${ }^{69}$

Despite these safety concerns, AOSLO fluorescence imaging of the in vivo retina is a suitable technique to be used in animal models, where different techniques to tag certain retinal cells with fluorescence molecules are available, and other retinal cell types beyond the photoreceptor mosaic or the RPE cells can be observed. ${ }^{70}$ In particular, ganglion cells have only been observed in vivo in retinas using exogenous fluorescence imaging techniques. In particular, primates were injected with a fluorescent dye in the lateral geniculate nucleus of the thalamus, and after allowing retrograde transport of the dye to the ganglion cells for a few days, images of the retina of the animal were acquired, and individual ganglion cells could be observed using AOSLO fluorescence imaging.

In the case of murine retinas, individual ganglion cells have been observed to study their function. For that, a gene that produced intense expression of calcium indicator has been delivered to mice retinas with intracranial injection of rabies vector. The response of this indicator to different exposures to light has been studied on these mice over days in order to study the function of ganglion cells. ${ }^{71}$

Other cellular structures such as microglia and pericites have also been observed in retinas of transgenic mice. ${ }^{72-74} \mathrm{By}$ labeling via transduction from retrograde viral vector, retinal cellular structure has been tagged in order to be imaged using fluorescence AOSLO. ${ }^{73}$

\section{Simultaneous AOSLO and OCT}

Over the past few decades, OCT has become one of the most important retinal imaging techniques to be used in the clinic. Its high axial resolution, of just a few microns, and high sensitivity are used to generate images of the different layers of the retina.

OCT systems have been successfully combined with AO in the past, showing impressive three-dimensional reconstruction images of the retina with unprecedented resolution, ie, below $3 \times 3 \times 3 \mu \mathrm{m}^{3} .{ }^{9}$ However, it has proved challenging to provide information on individual cells in the retina. ${ }^{75}$

Since the optical setups for AOSLO and Adaptive Optics Optical Coherence Tomography (AOOCT) systems are very similar, efforts have been devoted to build AO-assisted retinal imaging systems that can provide, simultaneously, SLO and OCT images of the fundus of the in vivo human eye. This is a very interesting option, since the system is able to provide two different images with pixel-to-pixel correspondence between them, which can complement the information they provide about the subject's retina. ${ }^{4,5}$ The main advantage of this implementation is that OCT images can benefit from high axial resolution compared to SLO ones, while the SLO image, which usually presents higher SNR, can be used as a reference in order to eliminate the effect of saccade and microsaccade eye movements on the OCT image. Using this approach, it has been possible to report the observation of individual cells in different retinal layers, such as the photoreceptor layer and RPE, but it has also been possible to observe differences in contrast on different layers of the retina, such as the external limiting membrane and inner and outer segments, which are still a challenge for AOSLO systems due to their limited axial resolution. ${ }^{39}$ Furthermore, the same technique has also been used to image the vasculature of the in vivo human retina, including vessel walls and individual erythrocytes inside the vessels. ${ }^{40}$

\section{Two-photon excitation fluorescence imaging}

As mentioned earlier, fluorescence imaging of the retina can be very useful, not only in the diagnosis of different retinal diseases using AF but also in research, since it allows for the observation of retinal features tagged with extrinsic fluorescent dyes. However, as it has also been pointed out and is further discussed in the "Safety issues" section, some concerns have arisen related to the use of blue light for AF excitation. ${ }^{76}$ Two-photon excitation fluorescence (TPEF) may provide a solution to these issues, since in TPEF the light source that is used for excitation is usually in the infrared area of the light spectrum, which is safer and more comfortable for the subject. TPEF has great potential to monitor retinoid changes in the human eye, since it could reveal details of molecular processes in the retina and RPE. Retinyl esters and all-trans-retinal condensation products are two types of retinoid fluorophores that are present in the retina and RPE. TPEF has been used in the past to understand metabolism, structure, signal transduction, and signal transmission in the eye. ${ }^{77}$

In TPEF microscopy, pulsed laser sources are traditionally used. Light pulses concentrate high peak power in short periods of time, where photon density can be very high, while keeping the average power low. Furthermore, high numerical objectives concentrate these pulses in space, and fluorescence is only generated in a small volume around the focal point, where the light is tightly focused and high photon densities needed for two photon absorption are reached. Due 
to this reason, TPEF microscopy provides optical sectioning capabilities, since fluorescence is only generated on the focal plane of the microscope objective.

In the case of in vivo applications, the numerical aperture (NA) is dominated by the eye, and it is therefore set to a value of NA $\sim 0.23$ in humans, which is a limiting factor to efficiently generate nonlinear fluorescence signal. Furthermore, just as in the case of fluorescence imaging, the power of the laser beam used to observe two-photon absorption in the retina is limited for the safety of the subject. For these reasons, the implementation of TPEF imaging techniques in vivo has proven challenging in both humans and animal models. Despite these limitations, AOSLO TPEF imaging has been implemented in the primate retina, showing the photoreceptor cone mosaic using TPEF. ${ }^{78}$ Exposure times used to generate these images were very long ( $>10$ minutes). In order to register AOTPEF images, the AOSLO images were used. Similar to what has been explained in the case of simultaneous AOOCT/SLO imaging, AOSLO and AOTPEF images present pixel-to-pixel correspondence, and AOSLO signal was used to register both reflectance AOSLO and AOTPEF images over the acquisition time. More recent studies provide an insight into what may be the potential of applying this technique in vivo in the human eye. ${ }^{76}$

Numerical aperture in the mouse eye, NA $\sim 0.49$, is two times greater than that of the human eye, and this translates into a maximum lateral resolution of $0.7 \mu \mathrm{m}$ in mice $(1.4 \mu \mathrm{m}$ in humans) and $6 \mu \mathrm{m}$ in the axial direction $(25 \mu \mathrm{m}$ for humans). Although murine eyes present high aberrations that have posed a challenge in the past, $\mathrm{AO}$ has been successfully used to correct them. ${ }^{72,79-81}$ All this indicates that the murine eye can be appropriate for the successful implementation of this technique. TPEF AOSLO has been used to image ganglion cells labeled with green fluorescent protein in the eyes of mice ${ }^{81}$ and also photoreceptor cells and endogenous and artificial fluorophores in retinas affected by genetic disorders, environmental stress, or drug therapy. ${ }^{82}$

\section{Image stabilization}

In point-scanning techniques such as SLO and OCT in which images are generated by scanning the retina point by point, saccadic and microsaccadic eye motion keeps the retina moving while the images are being acquired, introducing distortion. This is not a problem for techniques such as fundus cameras, in which the whole retinal image is acquired at once and in a very short period of time.

Eye motion is especially a problem in the case of AOSLO, in which applications are usually devoted to observe small areas in the retina, and motion effects may be comparable to the image size itself. Therefore, precise saccadic and microsaccadic motion correction is essential in order to provide high-quality and high-resolution retinal images.

There are several methodologies available to determine eye motion during image acquisition, and some of them have been implemented into AOSLO imaging systems. One of the most commonly used approaches to avoid eye motion effects is image post processing. ${ }^{83-85}$ Once an image sequence has been acquired over a period of time, specific software is used in order to register them, so that they can be averaged together to reduce noise and increase their SNR and quality. In the process of registration, information on the motion of the eye during image acquisition can be estimated and studied. As mentioned earlier, this information can also be very valuable to stabilize different imaging modalities that are recorded at once in the same multimodal imaging system, such is the case of TPEF AOSLO ${ }^{78}$ or AOSLO/OCT images. ${ }^{39}$

Image stabilization has also been implemented during acquisition of the images, using different methods, which rely on optical stabilization ${ }^{86,87}$ or digital registration. ${ }^{88}$ One of the main interests in correcting the eye motion during image acquisition is to enable psychophysical experiments where accurate control of the position of the retina that is stimulated is needed. These psychophysical studies have revealed how the motion of the retina can affect the way the brain processes visual stimuli, ${ }^{89}$ or how parameters such as photoreceptor size, ${ }^{90}$ or even disease ${ }^{15}$ can affect perception.

\section{Image analysis}

Image analysis is crucial in order to standardize the use of AOSLO in the clinic, and the interpretation of the information provided by this imaging technique should be very carefully analyzed..$^{91}$ It seems necessary that the information that AOSLO images may encode can be efficiently deciphered and related to health and disease indicators. These indicators probably will become more reliable and meaningful as the use of the technique increases.

The difference of these indicators has been suggested in order to characterize the physical distribution of photoreceptor cells over the retina. Voronoi analysis has been suggested as a method to analyze AOSLO images of cell mosaics. Using this method, the number of neighbors of each cell in the mosaic is determined according to their distance. This analysis has been used to provide quantitative information from AOSLO images, as it is a way to assess the regularity of individual cell mosaics. ${ }^{92-94}$ In addition, the link between cone reflectivity and photoreceptor health and 
function has been studied, although it has been shown that reflectivity cannot be related to photoreceptor health and that low reflecting cones on healthy subjects show normal function. ${ }^{95,96}$

However, up to date, one of the most widely used parameters chosen to objectively characterize the in vivo retina is the cone and rod density with eccentricity. ${ }^{97}$ Sometimes, photoreceptor spacing has been preferred rather than density, since it does not require to identify every single photoreceptor cell in an area in order to be calculated. ${ }^{91}$ Changes in the density of cone and rod photoreceptors with eccentricity from the fovea, and also the variability of this parameter across subjects, have been widely studied in the past. ${ }^{98-100}$ These experiments have allowed for the validation of the technique and have helped confirm that the features observed on the image are indeed photoreceptors. Histological measurement of photoreceptor density has been compared to information acquired across different AOSLO systems. ${ }^{12,101}$ This validation is extremely important in order to appropriately interpret novel imaging techniques, such as AOSLO, where new information is revealed. This parameter has been determined not only on healthy subjects but also on patients suffering from different diseases ${ }^{13,14,102-104}$ and has also been used to determine disease progression and even treatment efficacy. ${ }^{16}$ Finally, some methods have been developed in order to automatically detect the position of individual photoreceptor cells in AOSLO images, ${ }^{92,105,106}$ although they should be used carefully when assessing diseased retinas..$^{91}$

\section{Safety issues}

The reports of AOSLO often detail the laser power used, which is usually well below the safety limits dictated by laser safety regulations (or in general the American National Standards Institute's laser safety standards). These regulations describe what is called the maximum permissible exposure, the maximum laser power over a certain pupil area on the cornea that is safe to apply on the subject.

Some findings in the past few years have raised some concerns about these safety limits. These concerns were mainly triggered by the observation of changes in the AF signal in the RPE layer of primates after they were observed using AOSLO ${ }^{65,66}$ In these experiments, the RPE cell mosaic was imaged in vivo using a blue laser source. Although the amount of light used to perform these experiments was below the safety limits, a reduction in the AF signal was observed on those areas illuminated. Furthermore, these changes observed became permanent depending on the power used for illumination. These changes have also been observed in the case of human subjects when using infrared light sources, even when as in the previous case, the amount of light used was below the maximum permissible exposure. ${ }^{68}$

These concerns have triggered a debate on the safety limits for in vivo retinal imaging, especially since these techniques are ultimately aimed at imaging patients who suffer from different retinal diseases, and it may be possible that these retinas are more vulnerable to high light levels.

\section{Conclusion}

AOSLO technology has enabled very important achievements in the in vivo retinal imaging. Maybe, one of the most representative ones is the capability to show in vivo cell mosaics throughout the healthy and diseased retina over different retinal layers, such as the photoreceptor layer or the RPE. This feature has proven very interesting in the study of different retinal diseases; in the case of degenerative processes, it allows for very precise characterization of their progression, or even regeneration.

The versatility of AOSLO systems has allowed the development of many different applications beyond the study of individual photoreceptor cells, such as capillary vessels imaging through fluorescein angiography, RPE or NFL imaging through AF, and nonconfocal imaging, which greatly extends the possible applications of the technique to many other fields, such as diabetic retinopathy or glaucoma.

AOSLO technology is also helping to understand the process of vision through psychophysical experiments that can link the anatomy of the retina with the perception of the subject.

Furthermore, the study of animal models is also extending our knowledge of the retina and help ophthalmologists to study and understand chemical processes and function of different types of cells in vivo.

\section{Acknowledgments}

The authors acknowledge Dr J Monés (Institut de la Màcula i la Retina, Spain) and also Dr Johnny Tam (National Eye Institute, USA) for his contribution to Figure 3, Prof Alfredo Dubra (Medical College of Wisconsin, USA) for Figure 4, and Andres Guevara (University of Rochester, USA) for Figure 5. In addition, the authors acknowledge financial support from Laserlab-Europe (EU-H2020 654148), the Spanish MINECO (Severo Ochoa grant SEV-2015-0522) and the Generalitat de Catalunya and Biophotonic Plus initiative project LITE. This research has been partially supported by Fundació Cellex Barcelona and has been conducted at ICFO's Super Resolution Light Microscopy and Nanoscopy Facility. 


\section{Disclosure}

The authors report no conflicts of interest in this work.

\section{References}

1. Liang J, Williams DR, Miller DT. Supernormal vision and high-resolution retinal imaging through adaptive optics. J Opt Soc Am A Opt Image Sci Vis. 1997;14(11):2884-2892.

2. Zhang Y, Rha J, Jonnal RS, Miller D. Adaptive optics parallel spectral domain optical coherence tomography for imaging the living retina. Opt Express. 2005;13(12):4792-4811.

3. Zawadzki RJ, Jones SM, Olivier SS, et al. Adaptive-optics optical coherence tomography for high-resolution and high-speed 3D retinal in vivo imaging. Opt Express. 2005;13(21):8532.

4. Merino D, Dainty C, Bradu A, Podoleanu AG. Adaptive optics enhanced simultaneous en-face optical coherence tomography and scanning laser ophthalmoscopy. Opt Express. 2006;14(8):3345-3353.

5. Pircher M, Zawadzki RJ, Evans JW, Werner JS, Hitzenberger CK. Simultaneous imaging of human cone mosaic with adaptive optics enhanced scanning laser ophthalmoscopy and high-speed transversal scanning optical coherence tomography. Opt Lett. 2008;33(1): 22-24.

6. Roorda A, Williams DR. The arrangement of the three cone classes in the living human eye. Nature. 1999;397(6719):520-522.

7. Dubra A, Sulai Y, Norris JL, et al. Non-invasive in vivo imaging of the human rod photoreceptor mosaic using a confocal adaptive optics scanning ophthalmoscope. Biomed Opt Express. 2011;2(7): 1864-1876.

8. Dees EW, Dubra A, Baraas RC. Variability in parafoveal cone mosaic in normal trichromatic individuals. Biomed Opt Express. 2011;2(5): 1351-1358.

9. Miller DT, Kocaoglu OP, Wang Q, Lee S. Adaptive optics and the eye (super resolution OCT). Eye (Lond). 2011;25(3):321-330.

10. Miller DT, Williams DR, Morris GM, Liang J. Images of cone photoreceptors in the living human eye. Vision Res. 1996;36(8): 1067-1079.

11. Carroll J. Adaptive optics retinal imaging: applications for studying retinal degeneration. Arch Ophthalmol. 2008;126(6):857.

12. Merino D, Duncan JL, Tiruveedhula P, Roorda A. Observation of cone and rod photoreceptors in normal subjects and patients using a new generation adaptive optics scanning laser ophthalmoscope. Biomed Opt Express. 2011;2(8):2189-2201.

13. Zhang Y, Wang X, Rivero EB, et al. Photoreceptor perturbation around subretinal drusenoid deposits as revealed by adaptive optics scanning laser ophthalmoscopy. Am J Ophthalmol. 2014;158(3):584.e1-596.e1.

14. Menghini M, Lujan BJ, Zayit-Soudry S, et al. Correlation of outer nuclear layer thickness with cone density values in patients with retinitis pigmentosa and healthy subjects. Invest Ophthalmol Vis Sci. 2014;56(1):372-381.

15. Ratnam K, Carroll J, Porco TC, Duncan JL, Roorda A. Relationship between foveal cone structure and clinical measures of visual function in patients with inherited retinal degenerations. Invest Ophthalmol Vis Sci. 2013;54(8):5836-5847.

16. Talcott KE, Ratnam K, Sundquist SM, et al. Longitudinal study of cone photoreceptors during retinal degeneration and in response to ciliary neurotrophic factor treatment. Invest Ophthalmol Vis Sci. 2011; 52(5):2219-2226.

17. Horton JC, Parker AB, Botelho JV, Duncan JL. Spontaneous regeneration of human photoreceptor outer segments. Sci Rep. 2015;5: 12364.

18. Webb RH, Hughes GW, Pomerantzeff O. Flying spot TV ophthalmoscope. Appl Opt. 1980;19(17):2991-2997.

19. Webb RH. Confocal optical microscopy. Rep Prog Phys. 1996;59(3): $427-471$
20. Roorda A, Romero-Borja F, Donnelly III W, Queener H, Hebert T, Campbell M. Adaptive optics scanning laser ophthalmoscopy. Opt Express. 2002;10(9):405-412.

21. Romero-Borja F, Venkateswaran K, Roorda A, Hebert T. Optical slicing of human retinal tissue in vivo with the adaptive optics scanning laser ophthalmoscope. Appl Opt. 2005;44(19):4032-4040.

22. Navarro R, Losada MA. Aberrations and relative efficiency of light pencils in the living human eye. Optom Vis Sci. 1997;74(7):540-547.

23. Iglesias I, Ragazzoni R, Julien Y, Artal P. Extended source pyramid wavefront sensor for the human eye. Opt Express. 2002;10(9):419-428.

24. Díaz-Doutón F, Pujol J, Arjona M, Luque SO. Curvature sensor for ocular wavefront measurement. Opt Lett. 2006;31(15):2245-2247.

25. Liang J, Grimm B, Goelz S, Bille JF. Objective measurement of wave aberrations of the human eye with the use of a Hartmann-Shack wave-front sensor. J Opt Soc Am A Opt Image Sci Vis. 1994;11(7):1949-1957.

26. Wilson $\mathrm{T}$. The role of the pinhole in confocal imaging systems. In: Pawley JB, editor. Handbook of Biological Confocal Microscopy. Boston, MA: Springer; 1990:113-126. Available from: http://link. springer.com/10.1007/978-1-4615-7133-9_11. Accessed July 3, 2015.

27. Fienup JR, Miller JJ. Aberration correction by maximizing generalized sharpness metrics. J Opt Soc Am A Opt Image Sci Vis. 2003;20(4): 609-620.

28. Booth MJ. Adaptive optics in microscopy. Philos Trans A Math Phys Eng Sci. 2007;365(1861):2829-2843.

29. Hofer H, Sredar N, Queener H, Li C, Porter J. Wavefront sensorless adaptive optics ophthalmoscopy in the human eye. Opt Express. 2011; 19(15):14160-14171.

30. Wong KS, Jian Y, Cua M, Bonora S, Zawadzki RJ, Sarunic MV. In vivo imaging of human photoreceptor mosaic with wavefront sensorless adaptive optics optical coherence tomography. Biomed Opt Express. 2015;6(2):580-590.

31. Zhang Y, Poonja S, Roorda A. MEMS-based adaptive optics scanning laser ophthalmoscopy. Opt Lett. 2006;31(9):1268-1270.

32. Zou W, Qi X, Burns SA. Woofer-tweeter adaptive optics scanning laser ophthalmoscopic imaging based on Lagrange-multiplier damped least-squares algorithm. Biomed Opt Express. 2011;2(7):1986-2004.

33. Zou W, Burns SA. Testing of Lagrange multiplier damped leastsquares control algorithm for woofer-tweeter adaptive optics. Appl Opt. 2012;51(9):1198-1208.

34. Webb RH, Hughes GW, Delori FC. Confocal scanning laser ophthalmoscope. Appl Opt. 1987;26(8):1492-1499.

35. Gómez-Vieyra A, Dubra A, Malacara-Hernández D, Williams DR. First-order design of off-axis reflective ophthalmic adaptive optics systems using afocal telescopes. Opt Express. 2009;17(21): 18906-18919.

36. Burns SA, Tumbar R, Elsner AE, Ferguson D, Hammer DX. Largefield-of-view, modular, stabilized, adaptive-optics-based scanning laser ophthalmoscope. J Opt Soc Am A Opt Image Sci Vis. 2007;24(5): 1313-1326.

37. Dubra A, Sulai Y. Reflective afocal broadband adaptive optics scanning ophthalmoscope. Biomed Opt Express. 2011;2(6):1757-1768.

38. Felberer F, Kroisamer JS, Hitzenberger CK, Pircher M. Lens based adaptive optics scanning laser ophthalmoscope. Opt Express. 2012; 20(16):17297-17310.

39. Felberer F, Kroisamer JS, Baumann B, et al. Adaptive optics SLO/OCT for 3D imaging of human photoreceptors in vivo. Biomed Opt Express. 2014;5(2):439-456.

40. Felberer F, Rechenmacher M, Haindl R, Baumann B, Hitzenberger CK, Pircher M. Imaging of retinal vasculature using adaptive optics SLO/ OCT. Biomed Opt Express. 2015;6(4):1407.

41. Zayit-Soudry S, Duncan JL, Syed R, Menghini M, Roorda AJ. Cone structure imaged with adaptive optics scanning laser ophthalmoscopy in eyes with nonneovascular age-related macular degeneration. Invest Ophthalmol Vis Sci. 2013;54(12):7498-7509.

42. Meadway A, Wang X, Curcio CA, Zhang Y. Microstructure of subretinal drusenoid deposits revealed by adaptive optics imaging. Biomed Opt Express. 2014;5(3):713-727. 
43. Chen M, Chui TYP, Alhadeff $P$, et al. Imaging retinal nerve fiber bundles in glaucoma patients with deep local visual field damage of the macular region. Invest Ophthalmol Vis Sci. 2014;55(13):4778.

44. Hood DC, Chen MF, Lee D, et al. Confocal adaptive optics imaging of peripapillary nerve fiber bundles: implications for glaucomatous damage seen on circumpapillary OCT scans. Transl Vis Sci Technol. 2015;4(2):12.

45. Chen MF, Chui TYP, Alhadeff P, et al. Adaptive optics imaging of healthy and abnormal regions of retinal nerve fiber bundles of patients with glaucoma. Invest Ophthalmol Vis Sci. 2015;56(1):674-681.

46. Scoles D, Higgins BP, Cooper RF, et al. Microscopic inner retinal hyper-reflective phenotypes in retinal and neurologic disease. Invest Opthalmol Vis Sci. 2014;55(7):4015-4029.

47. Martin JA, Roorda A. Direct and noninvasive assessment of parafoveal capillary leukocyte velocity. Ophthalmology. 2005;112(12): 2219-2224.

48. Tam J, Tiruveedhula P, Roorda A. Characterization of single-file flow through human retinal parafoveal capillaries using an adaptive optics scanning laser ophthalmoscope. Biomed Opt Express. 2011;2(4):781-793.

49. Tam J, Roorda A. Speed quantification and tracking of moving objects in adaptive optics scanning laser ophthalmoscopy. J Biomed Opt. 2011;16(3):036002.

50. Zhong Z, Petrig BL, Qi X, Burns SA. In vivo measurement of erythrocyte velocity and retinal blood flow using adaptive optics scanning laser ophthalmoscopy. Opt Express. 2008;16(17):12746-12756.

51. Tam J, Martin JA, Roorda A. Noninvasive visualization and analysis of parafoveal capillaries in humans. Invest Ophthalmol Vis Sci. 2009; 51(3):1691-1698

52. Tam J, Dhamdhere KP, Tiruveedhula P, et al. Disruption of the retinal parafoveal capillary network in type 2 diabetes before the onset of diabetic retinopathy. Invest Ophthalmol Vis Sci. 2011;52(12):9257-9266.

53. Tam J, Dhamdhere KP, Tiruveedhula P, et al. Subclinical capillary changes in non-proliferative diabetic retinopathy. Optom Vis Sci. 2012; 89(5):E692-E703.

54. Roorda A, Zhang Y, Duncan JL. High-resolution in vivo imaging of the RPE mosaic in eyes with retinal disease. Invest Ophthalmol Vis Sci. 2007;48(5):2297-2303.

55. Sulai YN, Scoles D, Harvey Z, Dubra A. Visualization of retinal vascular structure and perfusion with a nonconfocal adaptive optics scanning light ophthalmoscope. J Opt Soc Am A Opt Image Sci Vis. 2014;31(3): 569-579.

56. Chui TY, Vannasdale DA, Burns SA. The use of forward scatter to improve retinal vascular imaging with an adaptive optics scanning laser ophthalmoscope. Biomed Opt Express. 2012;3(10): 2537-2549.

57. Scoles D, Sulai YN, Dubra A. In vivo dark-field imaging of the retinal pigment epithelium cell mosaic. Biomed Opt Express. 2013;4(9):1710-1723

58. Guevara-Torres A, Williams DR, Schallek JB. Imaging translucent cell bodies in the living mouse retina without contrast agents. Biomed Opt Express. 2015;6(6):2106-2119.

59. von Rückmann A, Fitzke FW, Bird AC. Distribution of fundus autofluorescence with a scanning laser ophthalmoscope. Br J Ophthalmol. 1995;79(5):407-412.

60. Morgan JI, Dubra A, Wolfe R, Merigan WH, Williams DR. In vivo autofluorescence imaging of the human and macaque retinal pigment epithelial cell mosaic. Invest Ophthalmol Vis Sci. 2009;50(3): 1350-1359.

61. Rossi EA, Rangel-Fonseca P, Parkins K, et al. In vivo imaging of retinal pigment epithelium cells in age related macular degeneration. Biomed Opt Express. 2013;4(11):2527-2539.

62. Scoles D, Gray DC, Hunter JJ, et al. In-vivo imaging of retinal nerve fiber layer vasculature: imaging - histology comparison. $B M C$ Ophthalmol. 2009;9:9.

63. Pinhas A, Dubow M, Shah N, et al. In vivo imaging of human retinal microvasculature using adaptive optics scanning light ophthalmoscope fluorescein angiography. Biomed Opt Express. 2013;4(8):1305-1317.
64. Dubow M, Pinhas A, Shah N, et al. Classification of human retinal microaneurysms using adaptive optics scanning light ophthalmoscope fluorescein angiography. Invest Ophthalmol Vis Sci. 2014; 55(3):1299-1309.

65. Morgan JI, Hunter JJ, Masella B, et al. Light-induced retinal changes observed with high-resolution autofluorescence imaging of the retinal pigment epithelium. Invest Ophthalmol Vis Sci. 2008; 49(8):3715-3729.

66. Morgan JI, Hunter JJ, Merigan WH, Williams DR. The reduction of retinal autofluorescence caused by light exposure. Invest Ophthalmol Vis Sci. 2009;50(12):6015-6022.

67. Hunter JJ, Morgan JI, Merigan WH, Sliney DH, Sparrow JR, Williams DR. The susceptibility of the retina to photochemical damage from visible light. Prog Retin Eye Res. 2012;31(1):28-42.

68. Masella BD, Williams DR, Fischer WS, Rossi EA, Hunter JJ. Longterm reduction in infrared autofluorescence caused by infrared light below the maximum permissible exposure. Invest Ophthalmol Vis Sci. 2014;55(6):3929-3938.

69. Chui TY, Dubow M, Pinhas A, et al. Comparison of adaptive optics scanning light ophthalmoscopic fluorescein angiography and offset pinhole imaging. Biomed Opt Express. 2014;5(4):1173-1189.

70. Rossi EA, Chung M, Dubra A, Hunter JJ, Merigan WH, Williams DR. Imaging retinal mosaics in the living eye. Eye. 2011;25(3):301-308.

71. Yin L, Geng Y, Osakada F, et al. Imaging light responses of retinal ganglion cells in the living mouse eye. J Neurophysiol. 2013;109(9): 2415-2421.

72. Biss DP, Sumorok D, Burns SA, et al. In vivo fluorescent imaging of the mouse retina using adaptive optics. Opt Lett. 2007;32(6):659-661.

73. Geng Y, Dubra A, Yin L, et al. Adaptive optics retinal imaging in the living mouse eye. Biomed Opt Express. 2012;3(4):715-734.

74. Schallek J, Geng Y, Nguyen H, Williams DR. Morphology and topography of retinal pericytes in the living mouse retina using in vivo adaptive optics imaging and ex vivo characterization. Invest Opthalmol Vis Sci. 2013;54(13):8237-8250.

75. Lombardo M, Serrao S, Devaney N, Parravano M, Lombardo G. Adaptive optics technology for high-resolution retinal imaging. Sensors. 2012; 13(1):334-366

76. Palczewska G, Golczak M, Williams DR, Hunter JJ, Palczewski K. Endogenous fluorophores enable two-photon imaging of the primate eye. Invest Ophthalmol Vis Sci. 2014;55(7):4438-4447.

77. Imanishi Y, Lodowski KH, Koutalos Y. Two-photon microscopy: shedding light on the chemistry of vision ${ }^{\dagger}$. Biochemistry. 2007;46(34): 9674-9684.

78. Hunter JJ, Masella B, Dubra A, et al. Images of photoreceptors in living primate eyes using adaptive optics two-photon ophthalmoscopy. Biomed Opt Express. 2010;2(1):139-148.

79. Alt C, Biss DP, Tajouri N, Jakobs TC, Lin CP. An adaptive-optics scanning laser ophthalmoscope for imaging murine retinal microstructure. In: Manns F, Söderberg PG, Ho A, editors. Ophthalmic Technologies $X X$. Vol. 7550. San Jose, CA: Proceedings of the SPIE; 2010:755019.

80. Geng Y, Greenberg KP, Wolfe R, et al. In vivo imaging of microscopic structures in the rat retina. Invest Ophthalmol Vis Sci. 2009; 50(12):5872-5879

81. Sharma R, Yin L, Geng Y, et al. In vivo two-photon imaging of the mouse retina. Biomed Opt Express. 2013;4(8):1285-1293.

82. Palczewska G, Dong Z, Golczak M, et al. Noninvasive two-photon microscopy imaging of mouse retina and retinal pigment epithelium through the pupil of the eye. Nat Med. 2014;20(7):785-789.

83. Stevenson SB, Roorda A. Correcting for miniature eye movements in high resolution scanning laser ophthalmoscopy. In: Manns F, Soederberg PG, Ho A, Stuck BE, Belkin M, editors. Ophthalmic Technologies XV. San Jose, CA: Proceedings of SPIE; 2005:145-151.

84. Vogel CR, Arathorn DW, Roorda A, Parker A. Retinal motion estimation in adaptive optics scanning laser ophthalmoscopy. Opt Express. 2006;14(2):487-497 
85. Faisan S, Lara D, Paterson C. Scanning ophthalmoscope retinal image registration using one-dimensional deformation fields. Opt Express. 2011;19(5):4157-4169.

86. Hammer DX, Ferguson RD, Bigelow CE, Iftimia NV, Ustun TE, Burns SA. Adaptive optics scanning laser ophthalmoscope for stabilized retinal imaging. Opt Express. 2006;14(8):3354-3367.

87. Yang Q, Zhang J, Nozato K, et al. Closed-loop optical stabilization and digital image registration in adaptive optics scanning light ophthalmoscopy. Biomed Opt Express. 2014;5(9):3174-3191.

88. Yang Q, Arathorn DW, Tiruveedhula P, Vogel CR, Roorda A. Design of an integrated hardware interface for AOSLO image capture and conetargeted stimulus delivery. Opt Express. 2010;18(17):17841-17858.

89. Arathorn DW, Stevenson SB, Yang Q, Tiruveedhula P, Roorda A. How the unstable eye sees a stable and moving world. J Vis. 2013;13(10): 22.

90. Rossi EA, Roorda A. The relationship between visual resolution and cone spacing in the human fovea. Nat Neurosci. 2010;13(2):156-157.

91. Roorda A, Duncan JL. Adaptive optics ophthalmoscopy. Ann Rev Vis Sci. 2015;1(1):19-50.

92. Li KY, Roorda A. Automated identification of cone photoreceptors in adaptive optics retinal images. J Opt Soc Am A Opt Image Sci Vis. 2007; 24(5):1358-1363.

93. Galli-Resta L, Novelli E, Kryger Z, Jacobs GH, Reese BE. Modelling the mosaic organization of rod and cone photoreceptors with a minimalspacing rule. Eur J Neurosci. 1999;11(4):1461-1469.

94. Shapiro MB, Schein SJ, De Monasterio FM. Regularity and structure of the spatial pattern of blue cones of macaque retina. J Am Stat Assoc. 1985;80(392):803-812.

95. Cooper RF, Dubis AM, Pavaskar A, Rha J, Dubra A, Carroll J. Spatial and temporal variation of rod photoreceptor reflectance in the human retina. Biomed Opt Express. 2011;2(9):2577-2589.

96. BruceKS,Harmening WM,Langston BR, Tuten WS, RoordaA, SincichLC. Normal perceptual sensitivity arising from weakly reflective cone photoreceptors. Invest Opthalmol Vis Sci. 2015;56(8):4431-4438.
97. Godara P, Wagner-Schuman M, Rha J, Connor TB Jr, Stepien KE, Carroll J. Imaging the photoreceptor mosaic with adaptive optics: beyond counting cones. Adv Exp Med Biol. 2012;723:451-458.

98. Curcio CA, Sloan KR, Kalina RE, Hendrickson AE. Human photoreceptor topography. J Comp Neurol. 1990;292(4):497-523.

99. Curcio CA, Sloan KR. Packing geometry of human cone photoreceptors: Variation with eccentricity and evidence for local anisotropy. Vis Neurosci. 1992;9:169-180.

100. Liu BS, Tarima S, Visotcky A, et al. The reliability of parafoveal cone density measurements. Br J Ophthalmol. 2014;98(8):1126-1131.

101. Zhang T, Godara P, Blanco ER, et al. Variability in human cone topography assessed by adaptive optics scanning laser ophthalmoscopy. Am J Ophthalmol. 2015;160(2):290.e1-300.e1.

102. Duncan JL, Zhang Y, Gandhi J, et al. High-resolution imaging with adaptive optics in patients with inherited retinal degeneration. Invest Ophthalmol Vis Sci. 2007;48(7):3283-3291.

103. Yoon MK, Roorda A, Zhang Y, et al. Adaptive optics scanning laser ophthalmoscopy images in a family with the mitochondrial DNA T8993C mutation. Invest Ophthalmol Vis Sci. 2008;50(4):1838-1847.

104. Duncan JL, Talcott KE, Ratnam K, et al. Cone structure in retinal degeneration associated with mutations in the peripherin/RDS gene. Invest Ophthalmol Vis Sci. 2011;52(3):1557-1566.

105. Chiu SJ, Toth CA, Bowes Rickman C, Izatt JA, Farsiu S. Automatic segmentation of closed-contour features in ophthalmic images using graph theory and dynamic programming. Biomed Opt Express. 2012;3(5):1127-1140.

106. Chiu SJ, Lokhnygina Y, Dubis AM, et al. Automatic cone photoreceptor segmentation using graph theory and dynamic programming Biomed Opt Express. 2013;4(6): 924-937.
Clinical Ophthalmology

\section{Publish your work in this journal}

Clinical Ophthalmology is an international, peer-reviewed journa covering all subspecialties within ophthalmology. Key topics include: Optometry; Visual science; Pharmacology and drug therapy in eye diseases; Basic Sciences; Primary and Secondary eye care; Patient Safety and Quality of Care Improvements. This journal is indexed on

Submit your manuscript here: http://www.dovepress.com/clinical-ophthalmology-journal

\section{Dovepress}

PubMed Central and CAS, and is the official journal of The Society of Clinical Ophthalmology (SCO). The manuscript management system is completely online and includes a very quick and fair peer-review system, which is all easy to use. Visit http://www.dovepress.com/ testimonials.php to read real quotes from published authors. 